ILL-(TH)-93-21

\title{
COMPOSITENESS, TRIVIALITY AND BOUNDS ON CRITICAL EXPONENTS FOR FERMIONS AND MAGNETS
}

\author{
Aleksandar KOCIĆ and John KOGUT \\ Loomis Laboratory of Physics, University of Illinois, Urbana, Il 61801
}

\begin{abstract}
We argue that theories with fundamental fermions which undergo chiral symmetry breaking have several universal features which are qualitatively different than those of theories with fundamental scalars. Several bounds on the critical indices $\delta$ and $\eta$ follow. We observe that in four dimensions the logarithmic scaling violations enter into the Equation of State of scalar theories, such as $\lambda \phi^{4}$, and fermionic models, such as Nambu-Jona-Lasinio, in qualitatively different ways. These observations lead to useful approaches for analyzing lattice simulations of a wide class of model field theories. Our results imply that $\lambda \phi^{4}$ cannot be a good guide to understanding the possible triviality of spinor $Q E D$.
\end{abstract}




\section{Introduction}

There are two classes of theories in the literature that are used to model the Higgs sector of the Standard Model $[1,2]$. One is based on the self-interacting $\phi^{4}$ scalar theory in which the Higgs is elementary. The other is based on strongly interacting constituent fermi fields in which the Higg's particle is a fermion-antifermion bound state. A recent proposal for the second realization uses Nambu-Jona-Lasinio(NJL) models in which composite scalars emerge as a consequence of spontaneous chiral symmetry breaking [2]. In four dimensions both types of models have a trivial continuum limit and are meaningful only as effective theories with a finite cutoff. This restriction places constraints on the low-energy parameters e.g. bounds on the masses. We wish to point out in this letter that triviality in the two models is realized in different ways. The differences between theories of composite and elementary mesons can be expressed in terms of the critical indices $\delta$ and $\eta$, and several inequalities and bounds on these indices will follow. These results should prove useful in theoretical, phenomenological and lattice simulation studies of a wide class of model field theories.

We begin with a few comments about the physics in each model. In a NJL model [3], as a result of spontaneous chiral symmetry breaking, the pion-fermion coupling is given by the Goldberger-Treiman relation $g_{\pi N}=M_{N} / f_{\pi}$, where $M_{N}$ is the fermion mass and $f_{\pi}$ is the pion decay constant. Being the wavefunction of the pion, $f_{\pi}$ determines its radius as well: $r_{\pi} \sim 1 / f_{\pi}$. The Goldberger-Treiman can then be written in the suggestive form $g_{\pi N} \sim M_{N} r_{\pi}$. Thus, the coupling between pions and fermions vanishes as the size of the pion shrinks to zero. The origin of triviality of the Nambu-Jona-Lasinio model is precisely the loss of compositeness of the mesons [3]. The force between the fermions is so strong that the constituents collapse onto one another producing pointlike mesons and a noninteracting continuum theory. In a self-interacting scalar theory, like $\phi^{4}$, the mesons are elementary and the reason for triviality is different [4,5]. At short distances the interaction is repulsive, so there is no collapse. The structure of the scalars, needed for the interaction to survive the continuum limit, should be built by weakly interacting bosons. In four dimensions, the short ranged $\lambda \phi^{4}$ interaction fails to provide a physical size for the mesons. It cannot be felt by the particles because of the short-distance repulsion - they cannot meet where collective behavior can set in and produce macroscopic fluctuations. In this way the cutoff remains the only scale and the continuum limit is trivial.

Consider two simple, soluble examples: the large- $N$ limits of the $O(N) \sigma$ - and the four-fermi model $[6,7]$. They exhibit a phase transition at finite coupling for $2<d<4$. Their critical exponents are given in Table 1. As is apparent from the Table, the two sets of critical indices evolve differently when $d$ is reduced below 4. At first glance this might be surprising since both models break the same symmetry spontaneously and one expects that they describe the same low energy physics. The purpose of this paper is to show that this difference between the critical exponents is generally valid, irrespective of the approximations employed. As a consequence of this it will be possible to establish a bound on the exponent $\delta$ which for scalar theories is $\delta \geq 3$, and for fermionic theories is $\delta \leq 3$. Although in four dimensions the two sets of exponents coincide, they are accompanied by logarithmic corrections due to scaling violations. It 
consequently the scaling violations have opposite signs in the two classes of theories. These bounds are a consequence of different realizations of symmetry breaking, the essential difference being the fact that for scalar theories mesons are elementary, while in the case of the chiral transition in fermionic theories, they are composite. The bounds on $\delta$ are just another way of expressing this difference in terms of universal quantities. Finally, we will discuss the implications of these results on triviality in both models in four dimensions.

Table 1

Leading order critical exponents for the spherical and four-fermi model

$\begin{array}{ccc}\text { exponent } & \sigma \text {-model } & \text { four-fermi } \\ \beta & \frac{1}{2} & \frac{1}{d-2} \\ \nu & \frac{1}{d-2} & \frac{1}{d-2} \\ \delta & \frac{d+2}{d-2} & d-1 \\ \gamma & \frac{2}{d-2} & 1 \\ \eta & 0 & 4-d\end{array}$

\section{Mass ratios and bounds on $\delta$}

To approach the problem, it is convenient to adopt a particular view of the phase transition [8]. Instead of the order parameter we will use mass ratios to distinguish the two phases. While the order parameter is a useful quantity to parametrize the phase diagram, the spectrum carries direct information about the response of the system in it's different phases and its form does not change in the presence of an external symmetry-breaking field. In what follows we will switch from magnetic to chiral notation without notice. The correspondence is: magnetic field $(h) \Leftrightarrow$ bare mass $(m)$; magnetization $(M) \Leftrightarrow$ chiral condensate $(<\bar{\psi} \psi>)$; longitudinal and transverse modes $\Leftrightarrow(\sigma, \pi) ; h \rightarrow 0 \Leftrightarrow$ chiral limit. Theories that treat scalars as elementary will be referred to as 'magnets' and those that give rise to composite mesons as a consequence of spontaneous chiral symmetry breaking will be refered to as 'fermions'.

Consider the effect of spontaneous symmetry breaking on the spectrum from a physical point of view. In the symmetric phase, there is no preferred direction and symmetry requires the degeneracy between longitudinal and transverse modes (chiral partners). Therefore, in the zero-field (chiral) limit the ratio $R=M_{T}^{2} / M_{L}^{2}=M_{\pi}^{2} / M_{\sigma}^{2} \rightarrow 1$. As the magnetic field (bare mass) increases, the ratio decreases (because of level ordering, $\sigma$ is always heavier then $\pi$ ). In the broken phase, however, the ratio vanishes in the chiral limit because the pion is a Goldstone boson. This time, the ratio clearly increases away from the chiral limit. 
dynamics. The value of the ratio at large $h$ is less sensitive to variations in the coupling. The qualitative behavior of the mass ratio is sketched in Fig.1. The important property of the mass ratio, in this context, is that its properties follow completely from the properties of the order parameter [8]. This, after all, comes as no surprise since both quantities, $M$ and $R$, contain the same physics and merely reflect two aspects of one phenomenon.

The essential ingredients are the Equation of State (EOS) and the Ward identity which follows from it.

$$
h_{a}=M_{a} M^{\delta-1} f\left(t / M^{1 / \beta}\right), \quad \chi_{T}^{-1}=h / M, \quad \chi_{L}^{-1}=\frac{\partial h}{\partial M}
$$

where $t$ is the usual reduced coupling (temperature). The second equation follows from the first upon differentiation. It is the Ward identity that ensures the Goldstone theorem. The ratio of susceptibilities is a function of the reduced variable, $t / M^{1 / \beta}$, only and is determined completely by the critical indices and the universal function $f(x)$. Elementary algebra shows that the ratio $\chi_{L} / \chi_{T}$ is a function of one variable only, namely $R(t, h)=R\left(h / t^{\Delta}\right)$ which explains the behavior suggested by Fig.1. At the critical point the order parameter scales as $M \sim h^{1 / \delta}$ The susceptibility ratio is just a logarithmic derivative, $R=\partial \ln M / \partial \ln h$.

$$
\frac{1}{R}=\frac{\chi_{L}^{-1}}{\chi_{T}^{-1}}=\delta, \quad t=0
$$

At the critical point it is independent of the symmetry breaking field. The exponent $\delta$ measures the relative strength of the transverse and longitudinal responses [8].

The plot of $R$ versus $h$ (Fig.1) with the critical isotherm is a "universal phase diagram" - any curve $R>1 / \delta$ belongs to the symmetric phase and $R<1 / \delta$ to the broken phase. In this way, the entire range of coupling constant maps onto the interval $[0,1]$. The critical coupling maps onto $1 / \delta$. In these units the size of the broken phase is $1 / \delta$ and of the symmetric phase $1-1 / \delta$.

To explain the relevance of these observations to the problem in question, we recall some facts about magnets and compare them to the chiral transition.

- Magnets: At zero magnetic field, the critical coupling (temperature) separates two regions: the lowtemperature (weak coupling) from the high-temperature (strong coupling) phase. Spontaneous symmetry breaking occurs at low temperatures. As the dimensionality is reduced towards the lower critical value $(d=2)$, the disordering effect of infrared fluctuations becomes more important and the broken (massless) phase shrinks. On the "universal phase diagram", Fig.2a, this implies that $1 / \delta$ decreases below its mean field value. Thus, in this case $\delta>3$.

- Fermions: For the chiral transition the same reasoning applies except that now the broken and symmetric phases are interchanged. Chiral symmetry breaking is a strong coupling phenomenon. Thus, in this case it is the symmetric phase that shrinks in lower dimensions. As is seen in Fig.2b, this implies that $1 / \delta$ increases, leading to $\delta<3$.

These are the bounds announced in the introduction. 
One way to determine the sign of the scaling violations in four dimensions is to proceed in the spirit of the $\epsilon$-expansion i.e. to approach four dimensions from below [9]. The transcription to the language of scaling violations is established by the replacement $\epsilon \rightarrow 1 / \log \Lambda$ in the limit $\epsilon \rightarrow 0$. Thus, the extension of the arguments made before for $d<4$ can be made by simply taking the limit $d \rightarrow 4$. In this way we anticipate that the two inequalities prevail and suggest that the scaling violations have different signs in the two theories.

The difference in the sign of the scaling violations in fermions and magnets has a simple explanation and lies at the root of the difference between the patterns of symmetry breaking in the two systems. Imagine that we fix the temperature to its critical value $T=T_{c}$ and approach the critical point $\left(T=T_{c}, h=0\right)$ in the $(T, h)$ plane from the large- $h$ region. The possible similarity of the two models is related to their symmetry. This is apparent in the chiral (zero field) limit where this symmetry is manifest. By going away from this limit chiral symmetry is violated and the two models differ.

Consider the behavior of the mass ratio for magnets in a strong magnetic field, away from the scaling region. In this regime, the temperature factor can be neglected and the hamiltonian describes free spins in an external field ( $H \rightarrow h \sum_{i} S_{i}$ ). The energy of longitudinal excitations is proportional to the field-squared, $\chi_{L}^{-1} \sim h^{2}$, while the transverse mass remains fixed by the symmetry, namely $\chi_{T}^{-1}=h / M$ for any value of $h$. The effect of the external field is to introduce a preferred direction and its increase results in amplification of the difference between the longitudinal and transverse dirrections. For large $h$ the ratio scales as $R \sim 1 / h$. Therefore, an increase in magnetic field reduces the ratio towards zero. The critical isotherm in this case bends down (Fig.3).

For fermions, the mesons are fermion-antifermion composites. Close to the chiral limit, they are collective. However, as the constituent mass increases, they turn into atomic states and the main contribution to the meson mass comes from the rest energy of its constituents. In the limit of infinite bare mass, interactions are negligible and $M \rightarrow 2 m$ regardless of the channel. Thus, outside of the scaling region, an increase in $m$ drives the ratio to 1 (Fig.3).

Thus, the scaling violations for magnetis and fermionis have opposite signs. They contain knowledge of the physics away from the chiral limit where the two models are quite different and these differences remain as small corrections close to the chiral limit.

To establish the connection between scaling violations and triviality, we introduce the renormalized coupling. It is a dimensionless low-energy quantity that contains information about the non-gaussian character of the theory. It is conventionally defined as [10]

$$
g_{R}=-\frac{\chi^{(n l)}}{\chi^{2} \xi^{d}}
$$

where the nonlinear susceptibility $\chi^{(n l)}$ is the zero-momentum projection of the connected four-point function

$$
\chi^{(n l)}=\frac{\partial^{3} M}{\partial h^{3}}=\int_{123}<\phi(0) \phi(1) \phi(2) \phi(3)>_{c}
$$

The normalization fators, $\chi=\int_{x}<\phi(0) \phi(x)>_{c}$ and $\xi^{d}$, in eq.(3) take care of the four fields and the three integrations. In a gaussian theory all higher-point functions factorize, so $g_{R}$ vanishes. Using the hyperscaling 


$$
g_{R} \sim \xi^{(2 \Delta-\gamma-d \nu) / \nu}
$$

where $\Delta=\beta+\gamma$ is the gap exponent. Being dimensionless, $g_{R}$ should be independent of $\xi$ if $\xi$ is the only scale. Thus, the validity of hyperscaling requires that the exponent must vanish. It implies the relation, $2 \Delta-\gamma-d \nu=0$, between the critical indices. In general, it is known that the following inequality [11] holds

$$
2 \Delta \leq \gamma+d \nu
$$

The exponent in the expression for $g_{R}$ is always non-positive, so that violations of hyperscaling imply that the resulting theory is non-interacting.

Above four dimensions, the exponents are gaussian $(\gamma=1, \Delta=3 / 2, \nu=1 / 2)$. In this case, it is easy to verify the above inequality: $3 \leq 1+d / 2$, which amounts to $d \geq 4$. In four dimensions most field theoretical models have mean-field critical exponents, but with logarithmic corrections that drive $g_{R}$ to zero. Scaling violations in any thermodynamic quantity propagate into the renormalized coupling and, according to eq.(6), these violations lead to triviality.

Instead of using the ideas of the $\epsilon$-expansion where scaling is always respected and where equalities between exponents hold, we will fix $d=4$ and compute the logarithmic corrections to the critical exponents. In order to focus on the problem in question, we analyze two simple models: $\phi^{4}$ and $(\bar{\psi} \psi)^{2}$ theories both in the large- $N$ limit. The results that will be discussed are completely general and the two models are chosen just to make the argument simple. The effective actions for the two models are $[12,13]$

$$
\begin{gathered}
V(M)=-\frac{1}{2} t M^{2}+\frac{\lambda}{4} \frac{M^{4}}{\log (1 / M)} \\
V(<\bar{\psi} \psi>)=-\frac{1}{2} t<\bar{\psi} \psi>^{2}+<\bar{\psi} \psi>^{4} \log (1 /<\bar{\psi} \psi>)
\end{gathered}
$$

This is the leading log contribution only. In the first example, it is clear how log-corrections lead to triviality. The logarithm can simply be thought of as coming from the running coupling - quantum corrections lead to the replacement $\lambda \rightarrow \lambda_{R}$. The vanishing of the renormalized coupling is then manifest from eq.(7a). In the case of fermions, eq.(7b), the details are completely different - the analogous reasoning would lead to an erroneous conclusion that the renormalized coupling increases in the infrared. In eq.(7b) the explicit coupling is absent from the fluctuating term - it is already absorbed in the curvature. Once the curvature is fixed, the effective coupling is independent of the bare one. The vanishing of the renormalized coupling here follows from the wave funciton renormalization constant $Z \sim 1 / \ln (1 /<\bar{\psi} \psi>)[13]$.

In both cases the renormalized coupling is obtained through the nonlinear susceptibility. For simplicity, we work in the symmetric phase where the odd-point functions vanish. The correlation length is related to the susceptibility by $\xi^{2}=\chi / Z$. For magnets the folowing relations hold

$$
\begin{gathered}
\chi^{(n l)} \sim \chi^{4} \frac{\lambda}{\ln (1 / M)}, \quad Z=1 \\
g_{R} \sim Z^{2} \frac{\lambda}{\ln (1 / M)} \sim \frac{1}{\ln (1 / M)}
\end{gathered}
$$


For fermions, on the other hand, we have

$$
\begin{gathered}
\chi^{(n l)} \sim \chi^{4} \ln (1 /<\bar{\psi} \psi>), \quad Z \sim \frac{1}{\ln (1 /<\bar{\psi} \psi>)} \\
g_{R} \sim Z^{2} \ln (1 /<\bar{\psi} \psi>) \sim \frac{1}{\ln (1 /<\bar{\psi} \psi>)}
\end{gathered}
$$

In this context the following point should be made. The nonlinear susceptibility is a connected four-point function for the composite $\bar{\psi} \psi$ field. The free fermionic theory is not gaussian in $\bar{\psi} \psi$, so even in free field theory $g_{R}$ does not vanish. The fact that $g_{R} \rightarrow 0$ near the critical point indicates that the resulting theory is indeed gaussian in the composite field which results in a free bosonic theory in the continuum.

The Equation of State (EOS) is obtained from the effective potential by simple differentiation. To make the connection with $\delta$, we take $t=0$. The critical EOS for the magnets is [12]

$$
h \sim \frac{M^{3}}{\log (1 / M)}
$$

This defines the exponent $\delta$. Because of the scaling violations, eq.(10) vanishes faster then a pure power. So the "effective" $\delta$ is bigger then its mean-field value. It is convenient to define an effective $\delta$ in the standard way either as $\tilde{\delta}=\partial \log h / \partial \log M$ or, equivalently, through the mass ratio. In any case, the above relation gives

$$
\tilde{\delta}=3+\frac{1}{\log (1 / M)}
$$

Thus, on the ratio plot, Fig.2a, the critical isotherm is no longer flat, but goes down, as the $h$-field (order parameter) increases. The result of eq.(11) is well known in the literature and has been obtained in the past using the $\epsilon$-expansion: $\delta=3+\epsilon$ [12], where the correspondence with eq.(10) is made after the replacement $1 / \epsilon \rightarrow \log$. Such corrections to $\delta$, as eq.(11), can never occur in the case of the chiral transition.

For the four-fermi model the critical EOS [14] reads,

$$
m \sim<\bar{\psi} \psi>^{3} \log (1 /<\bar{\psi} \psi>)
$$

Unlike scalar theories, the log's appear in the numerator - the right hand side in eq.(12) vanishes slower then the pure power and the "effective" $\delta$ is smaller then the (pure) mean-field value.

$$
\tilde{\delta}=3-\frac{1}{\log (1 /<\bar{\psi} \psi>)}
$$

This difference in the position of the logarithm, eqs. $(10,12)$ or, equivalently, the sign of the scaling violations, eqs. $(11,13)$, is generic for the two models. It is independent of the approximation and follows from the differences in the physics of the two systems.

It is easy to compute logarithmic corrections for other exponents [11]. It turns out that, with these corrections, the hyperscaling relations become strict inequalities for both systems, e.g. $d \nu>2-\alpha, \gamma / \nu<$ $2-\eta, \beta / \nu<1+\eta / 2$, etc.. This statement is equivalent to triviality. 
We have seen that the two different realizations of spontaneous symmetry breaking can be expressed simply in terms of universal quantities. In this way, many apparently complicated dynamical questions become transparent. In addition, some of our observations lead to practical applications - they can be used for extracting the properties of the continuum limit of theories with new fixed points, especially when clear theoretical ideas about the low-energy physics of the theory are missing. Of special importance is the knowledge of the position of the logarithms when triviality is studied on the lattice. It is extremely difficult to establish the presence of the logarithms for a finite system and to disentangle them from finite size effects. The bounds obtained in this paper establish some criteria in this direction as far as chiral transitions are concerned. Recently, they were proven to be decisive in studies of the chiral transition of $Q E D$ [15] and in establishing triviality of the NJL model in four dimensions by computer simulations [16].

The literature on fermionic $Q E D$ abounds with loose statements such as " $Q E D$ is ultimately trivial and reduces to $\lambda \phi^{4} "$. If $Q E D$ suffers from complete screening (the Moscow zero), then we expect the NJL model to describe its triviality. One result of this paper is that $\lambda \phi^{4}$ cannot be a good guide to fermionic $Q E D$ under any circumstances!

There are several physical implications that the two bounds on $\delta$ imply and we discuss some of them briefly below.

The wavefunction renormalization constant respects the Lehmann bound: $0 \leq Z \leq 1$. Roughly speaking, $Z$ is the probability that the scalar field creates a single particle from the vacuum. The limit $Z=1$ corresponds to a noninteracting theory whereas the compositeness condition, $Z=0$, sets an upper bound on the effective coupling [17]. The anomalous dimension $\eta$ determines the scaling of $Z$ in the critical region $Z \sim \xi^{-\eta}$. It describes the scaling of the correlation function in the massless limit: $D(x) \sim 1 /|x|^{d-2+\eta}$. Small $\eta$ is associated with weak coupling and $\eta=O(1)$ with the strong coupling limit of the theory. It is related to the exponent $\delta$ through the hyperscaling relation

$$
\delta=\frac{d+2-\eta}{d-2+\eta}
$$

In the absence of an anomalous dimension, $\delta$ tends to grow above 3 as the dimensionality is reduced below $d=4$. In magnets this tendency is respected even for $\eta \neq 0$ as a consequence of the bound $\delta>3$. In all known examples of magnets with continuous symmetry breaking, $\eta$ remains small suggesting that the continuum theory is in some sense weakly interacting. For example, the large- $N$ expansion gives $\eta=O(1 / N)$ $[6]$.

The fermion situation is quite different. As a consequence of the bound $\delta<3$, the tendency of $\delta$ to grow in lower dimensions is reversed. To achieve this it is necessary that anomalous dimensions are large, $\eta=O(1)$, in order to counter the $d-2$ factor in the denominator of eq.(14). For example, in the large- $N$ expansion of four-fermi theories (below 4 dimensions), $\eta=4-d[7]$. Thus, fermionic theories are always strongly interacting because scalars couple strongly to the constituent fermions. However, in the context of the $1 / N$ expansion, it should be noted that in four-fermi theories, mesons don't interact among themselves at leading order. The $\sigma \pi \pi$ coupling is $O(1 / N)$ as for magnets.

We have outlined in the introduction the basic difference between the physics of triviality in two models: in the case of fermions, triviality is a consequence of having an interaction at short distances that is too 
especially visible from the behavior of the correlation functions in the two theories. In the scalar theory, where anomalous dimensions are small, the scaling of the correlation functions is weakly affected at short distances. They behave almost like free particle propagators, $D(x) \sim 1 /|x|^{d-2}$. For fermions, however, there are nontrivial changes in scaling due to strongly interacting dynamics at short distances. In four-fermi theory, for example, at large- $N \eta=4-d$ and $D(x) \sim 1 /|x|^{2}$ irrespective of $d$.

Another consequence of the difference in the bounds on $\delta$ concerns the physics in the broken phase. As an effect of spontaneous symmetry breaking a trilinear coupling is generated and the decay $\sigma \rightarrow \pi \pi$ is a dominant decay mode in the Goldstone phase. The pole in the $\sigma$ propagator is buried in the continuum states and the pion state saturates all the correlation functions. This is especially visible in lower dimensions and persists even at finite $h$. For fermions, however, this is not necessarily the case for the simple reason that the $\pi-\sigma$ mass-squared ratio in the broken phase is bounded by $1 / \delta>1 / 3$, away from the chiral limit and for the $\sigma \rightarrow \pi \pi$ decay to occur the masses must satisfy $M_{\pi}^{2} / M_{\sigma}^{2} \leq 1 / 4$. Thus, for appropriately chosen couplings and bare fermion masses, the decay becomes kinematically forbidden even in the broken phase.

Regarding the usage of perturbation theory, the rules are different for magnets and fermions. The applicability of perturbation theory to magnets was noted long ago [18]. Its success near two and four dimensions is not surprising. Below four dimensions, $\phi^{4}$ posesses an infrared fixed point at coupling $g_{c} \sim O(\epsilon)$. This coupling is an upper bound on the renormalized coupling i.e. $g_{R} \sim \epsilon$. So, the $\epsilon$-expansion is in effect renormalized perturbation theory. The critical exponents receive corrections of the type $\delta=3+O\left(g_{R}\right), \beta=$ $1 / 2+O\left(g_{R}\right)$, etc.. Thus, in $\phi^{4}$ the success of perturbation theory is a consequence of the fact that the infrared fixed point moves to the origin as $d \rightarrow 4$. In the non-linear $\sigma$-model the critical coupling is an ultraviolet fixed point that moves to the origin as $d \rightarrow 2$. The weak coupling phase is at low-temperatures. Due to the presence of Goldstone bosons, all the correlation functions are saturated with massless states and the entire low-temperature phase is massless. Every point is a critical point in the limit of vanishing magnetic field. Thus, the low-temperature expansion is an expansion in powers of $T$. Terms of the form $\exp (-M / T)$ are absent and there is no danger that they will be omitted by using perturbation theory. In this way, in principle, the critical region can be accessed through perturbation theory [18]. Clearly, such reasoning can not be applied to fermions simply because the weak coupling phase is symmetric. Thus, no matter how small the coupling is, perturbation theory omits the Goldstone physics as a matter of principle. It can not produce bound states that accompany the chiral transition and its applicability is questionable in general. Especially, it is difficult to imagine how perturbation theory could give a mass ratio that is constant, independent of the bare parameters, once the bare coupling is tuned to the critical value. Even if this were possible, the renormalized coupling would be sensitive to the variation of the bare mass leading to conflicting renormalization group trajectories as a consequence.

Finally, we comment on one possible use of the $\delta<3$ bound for controlling finite size effects in lattice studies of chiral transitions. As was argued in [8], it is convenient to introduce a plot $\chi_{\pi}^{-1}$ versus $<\bar{\psi} \psi>^{2}$. The usefulness of this plot becomes clear if we write the critical EOS. From $m \sim<\bar{\psi} \psi>^{\delta}$ and $\chi_{\pi}^{-1}=m /<$ $\bar{\psi} \psi>$, it follows that

$$
\chi_{\pi}^{-1} \sim\left(<\bar{\psi} \psi>^{2}\right)^{(\delta-1) / 2}
$$


concave downwards. On a small lattice the order parameter is smaller and pion mass is bigger then in the thermodynamic limit. Thus, small volume distortions are always in the direction of opposite concavity of the plot and the wrong concavity of this plot is a clear sign of the presence of finite size effects [8].

Acknowledgement We wish to acknowledge the discussions with E. Fradkin, A. Patrascioiou and E. Seiler. This work is supported by NSF-PHY 92-00148. 


\section{References}

[1] See for example The Standard Model Higgs Boson, Edited by M. Einhorn, (North-Holland, Amsterdam, 1991).

[2] Y. Nambu, in New Trends in Physics, proceedings of the XI International Symposium on Elementary Particle Physics, Kazimierz, Poland, 1988, edited by Z. Ajduk S. Pokorski and A. Trautman (World Scientific, Singapore, 1989); V. Miransky, M. Tanabashi and K. Yamawaki, Mod. Phys. Lett. A4, 1043 (1989); W. Bardeen, C. Hill and M. Lindner, Phys. Rev. D41, 1647 (1990).

[3] Y. Nambu and G. Jona-Lasinio, Phys. Rev. 122, 345 (1961).

[4] M. Aizenman, Comm. Math. Phys. 86, 1 (1982); C. Aragão de Carvalho, S. Caracciolo and J. Fröhlich, Nuc. Phys. B215[FS7], 209 (1983).

[5] M. Lüscher and P. Weisz, Nuc. Phys. B290[FS20], 25 (1987)

[6] S. K. Ma, in Phase Transitions and Critical Phenomena Vol.6, eds. C. Domb and M. Green (Academic Press, London, 1976).

[7] S. Hands, A. Kocić and J. B. Kogut, Phys. Lett. B273 (1991) 111.

[8] A. Kocić, J. B. Kogut and M.-P. Lombardo, Nuc. Phys. B398, 376 (1993).

[9] K. Wilson and J. Kogut, Phys. Rep. 12C, 75 (1974).

[10] See, for example, C. Itzykson and J.-M. Drouffe, Statistical Field Theory (Cambridge University Press, 1989); V. Privman, P.C. Hohenberg and A. Aharony, in Phase Transitions and Critical Phenomena Vol.14, eds. C. Domb and J.L. Lebowitz (Academic Press, London, 1991).

[11] B. Freedman and G. A. Baker Jr, J. Phys. A15 (1982) L715; R. Schrader, Phys. Rev. B14 (1976) 172; B. D. Josephson, Proc. Phys. Soc. 92 (1967) 269, 276.

[12] E. Brezin, J.-C. Le Guillou and J. Zinn-Justin, in Phase Transitions and Critical Phenomena Vol.6, eds. C. Domb and M. Green (Academic Press, London, 1976).

[13] T. Eguchi, Phys. Rev. D17, 611 (1978).

[14] S. Hands, A. Kocić and J. B. Kogut, Ann. Phys. 224, 29 (1993).

[15] A. Kocić, J. B. Kogut and K. C. Wang, Nucl. Phys. B398 (1993) 405.

[16] S. Kim, A. Kocić and J. Kogut (unpublished)

[17] S. Weinberg, Phys. Rev. 130, 776 (1963).

[18] E. Brezin and J. Zinn-Justin, Phys. Rev. B14, 3110 (1976). 


\section{Figure captions}

1. Susceptibility ratio as a function of magnetic field (bare mass) for fixed values of the temperature (coupling).

2. The behavior of the critical mass ratio, $R(t=0, h)=1 / \delta$, for different values of $d$, in a) magnets and $\mathrm{b}$ ) in the case of chiral transition.

3. Critical mass ratio, $R(t=0, h)$, for fermions and magnets in four dimensions over extended range of magnetic field (mass). 
This figure "fig1-1.png" is available in "png" format from: http://arxiv.org/ps/hep-th/9312007v1 
This figure "fig1-2.png" is available in "png" format from: http://arxiv.org/ps/hep-th/9312007v1 
This figure "fig1-3.png" is available in "png" format from: http://arxiv.org/ps/hep-th/9312007v1 